T.Б. НИкИТИНА, д-р техн. наук, проф., НТУ "ХПИ", В.В. КОЛОМИЕЦ, канд. техн. наук, доц., НТУ "ХПИ", М.О. ТАТАРЧЕНКО, асп., НТУ "ХПИ", В.В. ХОМЕНКО, студент, НТУ "ХПИ"

\title{
РАЗРАБОТКА И ЭКСПЕРИМЕНТАЛЬНОЕ ИССЛЕДОВАНИЕ СТЕНДА СТОХАСТИЧЕСКОЙ ДВУХМАССОВОЙ ЭЛЕКТРОМЕХАНИЧЕСКОЙ СИСТЕМЫ
}

Разработан стенд стохастической двухмассовой электромеханической системы. Проведены экспериментальные исследования динамических характеристик стенда стохастической двухмассовой электромеханической системы с типовым и робастным регуляторами. Показано, что система с робастным регулятором имеет меньшую ошибку по сравнению с системой с типовым регулятором. Ил.: 6. Библиогр.: 9 назв.

Ключевые слова: стенд стохастической двухмассовой электромеханической системы, экспериментальные исследования, динамические характеристики.

Введение. Для создания систем управления сложными многомассовыми электромеханическими системами с упругими и нелинейными элементами необходима математическая модель, адекватно описывающая объект управления. Достоверность результатов, полученных в ходе синтеза таких систем управления, может быть проверена только при работе с реальным объектом. Одним из подходов к решению данной проблемы является использование физических моделей - стендов, повторяющих структуру объекта управления. При этом первоначальная отладка регуляторов и сравнение их эффективности работы в условиях, максимально приближенных к реальным, выполняется на таком стенде, содержащем все необходимые элементы.

Постановка проблемы. Анализ последних достижений и публикаций по данной проблеме. Характерным режимом работы многих систем управления является отработка случайных задающих воздействий либо компенсация случайных внешних возмущающих воздействий широкого спектра частот [1]. В последнее время интенсивно развивается теория стохастического робастного управления [2 - 7], поэтому вопросы проектирования систем управления, работающих при случайных задающих и возмущающих воздействиях, являются актуальными. Для отладки алгоритмов управления такими системами необходим стенд, имитирующий работу реальной системы при отработке

(ㄱ Т.Б. Никитина, В.В. Коломиец, М.О. Татарченко, В.В. Хоменко, 2013 
случайных задающих воздействий либо компенсации случайных внешних возмущающих воздействий.

Исследовательские стенды для отладки программного обеспечения систем управления преобразователями с учетом особенностей кинематических связей между приводным двигателем и рабочим механизмом выпускаются многими электротехническими фирмами. В работах $[1,8,9]$ рассмотрены вопросы разработки стенда двухмассовой электромеханической системы и методики экспериментального исследования робастных систем управления главными приводами прокатных станов на этом стенде. Рассмотрим разработку аналогичного стенда двухмассовой электромеханической системы для экспериментального исследования эффективности стохастического робастного управления при случайных внешних воздействиях.

Цель работы. Разработка и изготовление исследовательского стенда стохастической двухмассовой электромеханической системы, позволяющего выполнить отладку программного обеспечения алгоритмов управления и исследование динамических характеристик стенда двухмассовой электромеханической системы с различными типами регуляторов при случайных внешних воздействиях.

Изложение материала исследования, полученных научных результатов. Вначале рассмотрим вопросы синтеза стохастической системы робастного управления, с помощью которой минимизируется анизотропия проектируемой системы, следуя работам [1 - 3]. Регулятор формирует управляющее воздействие на вход системы по ее измеряемому выходу и представляет собой динамический блок типа компенсатора, объединяющий стохастический робастный наблюдатель и стохастический робастный регулятор. Обозначим $A, B, C, D$ реализацию исходной системы, замкнутой этим регулятором, в следующем виде

$$
\mathfrak{I}\left(W, W_{k y}\right) \sim\left[\begin{array}{cc}
\bar{A} & \bar{B} \\
\bar{C} & D_{11}
\end{array}\right] \equiv\left[\begin{array}{cc|c}
A & B_{2} C & B_{1} \\
B C_{2} & \left.A^{A}\right) & B D_{21} \\
\hline C_{1} & D_{12} C & D_{11}
\end{array}\right] .
$$

Рассмотрим уравнение Риккати

$$
R=\bar{A}^{T} R \bar{A}+q \bar{C}^{T} \bar{C}+L^{T} \Sigma^{-1} L
$$

где $\sum \equiv\left[I_{m_{1}}-q D_{11}^{T} D_{11}-\bar{B}^{T} R \bar{B}\right]^{-1}$;

$$
L \equiv\left[L_{1} \quad L_{2}\right] \equiv \sum\left[\bar{B}^{T} R \bar{A}+q D_{11}^{T} \bar{C}\right]
$$


Если это уравнение Риккати имеет решение, то анизотропия сигнала равна

$$
-\frac{1}{2} \ln \operatorname{det}\left[\frac{m_{1} \sum}{\operatorname{tr}\left\{L P L^{T}+\Sigma\right\}}\right]=a,
$$

а эквивалентный формирующий фильтр

$$
W_{\phi} \sim\left[\begin{array}{cc}
\bar{A}+\bar{B} L & \bar{B} \Sigma^{1 / 2} \\
L & \Sigma^{1 / 2}
\end{array}\right]=\left[\begin{array}{cc|c}
A+B_{1} L_{1} & B_{1} L_{2}+B_{2} C & )^{B_{1} \Sigma^{1 / 2}} \\
{ }_{B}\left[C_{2}+D_{21} L_{1}\right] & A+B D_{21} L_{2} & B D_{21} \Sigma^{1 / 2} \\
\hline L_{1} & L_{2} & \Sigma^{1 / 2}
\end{array}\right],
$$

имеет грамиан управляемости, определяемый уравнением Ляпунова

$$
P=[\bar{A}+\bar{B} L] P[\bar{A}+\bar{B} L]^{T}+\bar{B} \sum \bar{B}^{T} .
$$

При этом $a$ - анизотропийная норма системы, замкнутой таким регулятором, равна

$$
\left\|\mathfrak{I}\left(W, W_{k y}\right)\right\|_{a}=\left\{\frac{1}{q}\left[1-\frac{m_{1}}{\operatorname{tr}\left\{L P L^{T}+S\right\}}\right]\right\}^{1 / 2} .
$$

Рассмотрим уравнение Риккати

$$
S=\left[A+B_{1} L_{1}\right] S\left[A+B_{1} L_{1}\right]^{T}+B_{1} \sum B_{1}^{T}-\Lambda \Theta \Lambda^{T},
$$

где $\Theta \equiv\left[C_{2}+D_{21} L_{1}\right] S\left[C_{2}+D_{21} L_{1}\right]^{T}+D_{21} \sum D_{21}^{T}$;

$$
\Lambda \equiv\left[\left[A+B_{1} L_{1}\right] S\left[C_{2}+D_{21} L_{1}\right]^{T}+B_{1} \sum D_{21}^{T}\right] \Theta^{-1} .
$$

Рассмотрим также уравнение Риккати

$$
T=\underline{A}^{T} T \underline{A}^{T}+\underline{C}^{T} \underline{C}-N^{T} \Pi \mathrm{N},
$$

где $\Pi \equiv \underline{B}^{T} T \underline{B}+D_{12}^{T} D_{12}, N \equiv\left[\begin{array}{ll}N_{1} & N_{2}\end{array}\right] \equiv-\Pi^{-1}\left(\underline{B}^{T} T \underline{A}+D_{12}^{T} \underline{C}\right)$,

в котором матрицы $\mathbf{A}, \mathbf{B}, \mathbf{C}, \mathbf{D}$ реализации имеют следующий вид

$$
\left[\begin{array}{cc}
\underline{A} & \underline{B} \\
\underline{C} & \underline{D}
\end{array}\right] \equiv\left[\begin{array}{cc|c}
A & B_{1} M & B_{2} \\
0 & A+B_{1} M+B_{1} C & 0 \\
\hline C_{1} & D_{11} M & \underline{D}
\end{array}\right] .
$$


Откуда может быть получена $\stackrel{A}{A}, \dot{B}, \stackrel{L}{C}, \dot{D}$ реализация регулятора, минимизирующего анизотропийную норму:

$$
\begin{aligned}
& \stackrel{)}{A}=B_{2} C+\left[I_{n}-\Lambda\right]\left[\begin{array}{cc}
A & B_{1} \\
C_{2} & D_{21}
\end{array}\right]\left[\begin{array}{l}
I_{n} \\
M
\end{array}\right], \\
& \hat{B}=\Lambda \text {, } \\
& \stackrel{\prime}{C}=N_{1}+N_{2} \text {. }
\end{aligned}
$$

Решение задачи синтеза анизотропийного регулятора сводится к вычислению трех алгебраических уравнений Риккати (1), (4), (5), уравнения Ляпунова (3) и уравнения специального вида (2) для вычисления уровня анизотропии входного сигнала. Для решения уравнений Риккати (1), (4), (5) используется алгоритм для нахождения обобщенных собственных векторов Шура, а для решения уравнения Ляпунова (3) используется алгоритм Шура для унитарной триангуляции матриц.

Описание стенда стохастической двухмассовой системы. На рис. 1 показана схема изучаемой системы. Механическая часть стенда выполнена на базе двух однотипных микродвигателей постоянного тока ДПТ-25-Н2. Преобразователем электрической энергии в механическую является микродвигатель Д1, а микродвигатель Д2 формирует величину нагрузки для Д1. Валы двигателей Д1 и Д2 соединены упругой передачей. Внешний вид стенда показан на рис. 2.

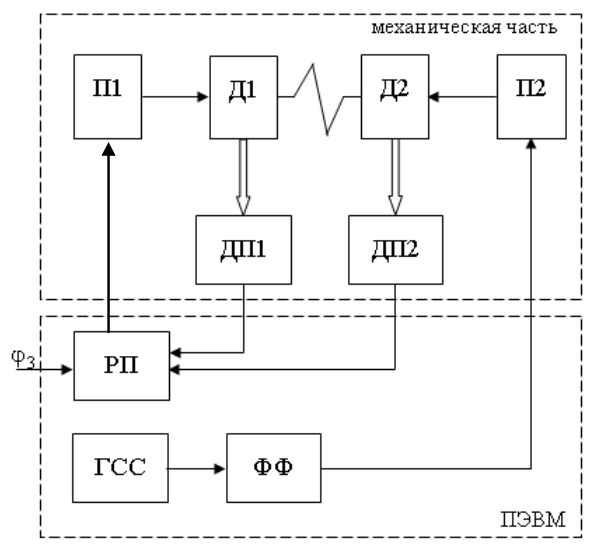

Рис. 1. Схема системы управления стендом в режиме регулирования углового положения 
Управление первым двигателем осуществляется от преобразователя П1 с помощью регулятора положения РП первого двигателя по сигналу с датчиков положения ДП1 либо ДП2 первого либо второго двигателей. С помощью второго двигателя создается момент нагрузки. Для имитации случайного воздействия на систему на вход второго двигателя подается случайный сигнал с выхода формирующего фильтра ФФ в виде колебательного звена. На вход формирующего фильтра подается сигнал типа белого шума от генератора случайных сигналов ГСС. Характеристики случайного изменения момента нагрузки определяются параметрами формирующего фильтра и собственно двигателя. Внешний вид системы управления стендом показан на рис. 3. На рис. 4 показан внешний вид платы системы управления стендом, а на рис. 5 показан внешний вид рабочего места оператора.

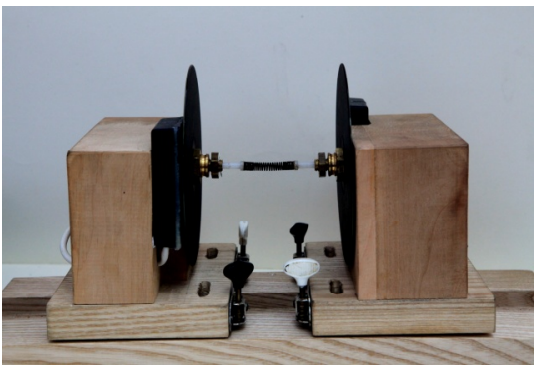

Рис. 2. Внешний вид стенда

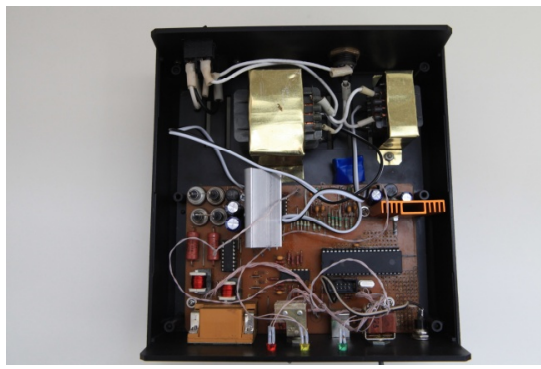

Рис. 4. Плата системы управления стендом

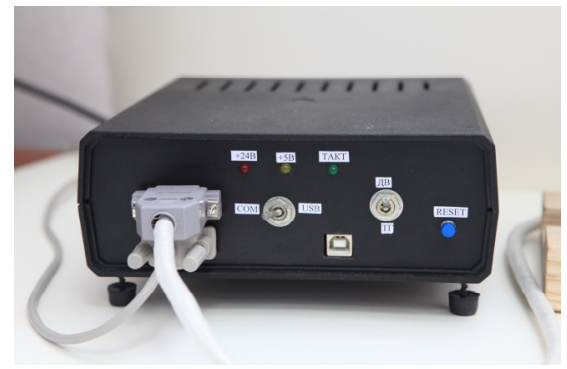

Рис. 3. Внешний вид системы управления стендом

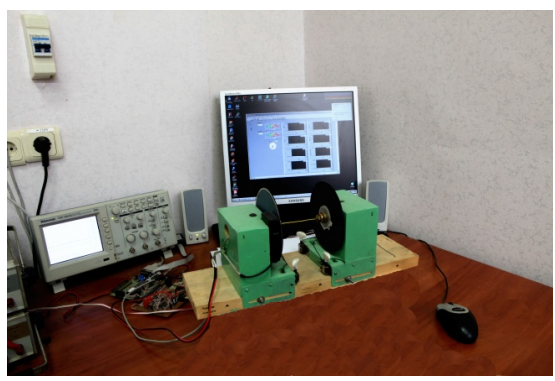

Рис. 5. Схема рабочего места оператора 
В режиме стабилизации, когда система замкнута по углу поворота, управление первым двигателем осуществляется от преобразователя П1 с помощью регулятора положения РП первого двигателя по сигналу с датчиков положения ДП1 либо ДП2 первого либо второго двигателей.

В режиме наведения, когда система замкнута по скорости изменения угла поворота, управление первым двигателем осуществляется от преобразователя П1 с помощью регулятора скорости РС первого двигателя по сигналу с датчиков скорости ДС1, ДС2 первого либо второго двигателей (на рис. 1 регулятор скорости РС и датчики скорости ДС1, ДС2 не показаны). Скорости вращения двигателей измеряются с помощью тех же импульсных датчиков положения ДП1, ДП2 первого и второго двигателей.

Экспериментальное исследование стенда при случайных входных сигналах. Рассмотрим работу системы при случайных входных сигналах. Для имитации случайного воздействия на систему на вход второго двигателя подается случайный сигнал с выхода формирующего фильтра. С помощью такой системы на вторую массу действует случайное изменение момента, формируемого с помощью формирующего фильтра и собственно второго двигателя. В качестве примера рассмотрена работа системы в режиме регулирования скорости при замыкании обратной связи по скорости второй массы. На рис. 6 показаны реализации переменных состояния системы с робастным регулятором, который замкнут по скорости вращения второй массы. На этом рисунке показаны следующие переменные состояния: а) и б) напряжения на первом и втором двигателях; в) и г) - скорости вращения первого и второго двигателей; $\partial$ ) и $e$ ) - токи якорных цепей первого и второго двигателей; ж) - момент упругости и з) - ошибка отработки заданного значения скорости первой массой. Как показали экспериментальные исследования, применение робастных регуляторов при случайном изменении момента нагрузки позволяет уменьшить ошибку регулирования скорости вращения двухмассовой электромеханической системы по сравнению с системой с типовыми регуляторами.

Выводы из проведенного исследования, перспективы этого направления. Разработана схема, техническое и программное обеспечение стенда стохастической двухмассовой электромеханической системы. Приведены результаты сравнения динамических характеристик системы управления стендом двухмассовой электромеханической системы с типовыми и робастными регуляторами при случайном 
изменении внешних воздействий. Показано, что применение стохастического робастного управления стендом двухмассовой электромеханической системы при случайном изменении момента нагрузки позволяет уменьшить ошибку регулирования по сравнению с системой с типовыми регуляторами в $1,5-2,1$ раза.



a)



в)

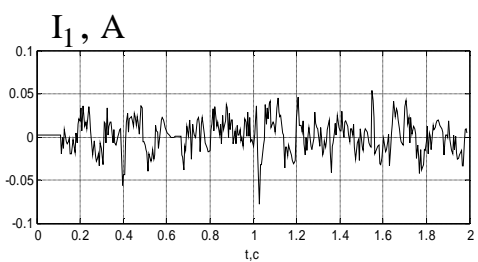

d)

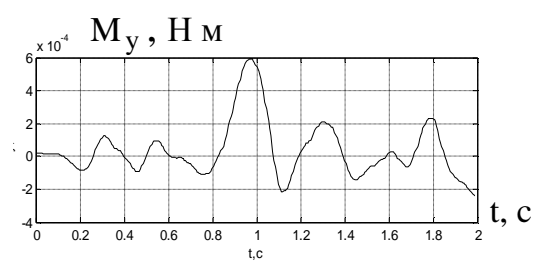

भ)

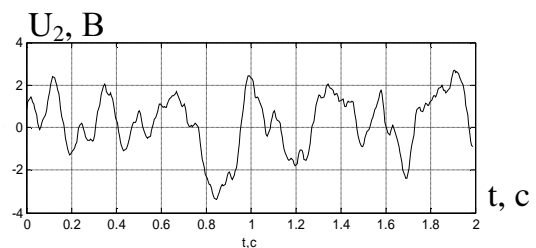

б)

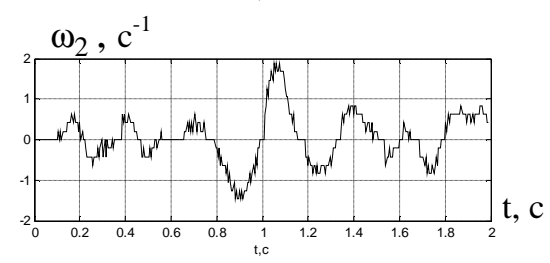

2)

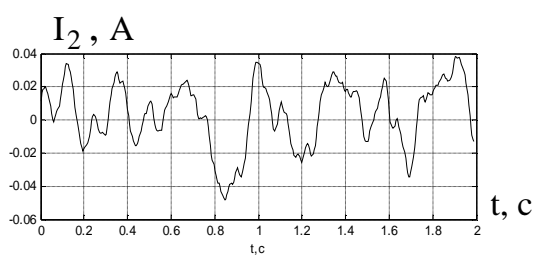

e)

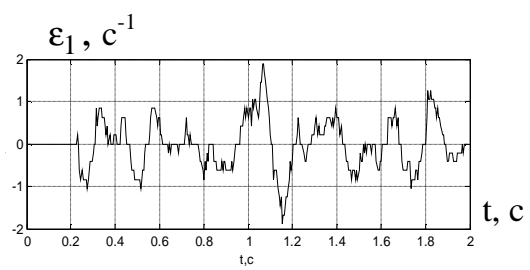

3)

Рис. 6. Реализации случайных процессов переменных состояния стенда стохастической двухмассовой системы

Список литературы: 1. Никитина Т.Б. Многокритериальный синтез робастного управления многомассовыми системами. - Монография / Т.Б. Никитина. - Харьков: ХАДУ, 


\section{ISSN 2079-0031 Вестник НТУ "ХПИ", 2013, № 19 (992)}

2013. - 432 с. 2. Никитина Т.Б. Синтез анизотропийных регуляторов многоканальных систем регулирования геометрических параметров проката / Т.Б. Никитина // Вісник Національного технічного університету "Харківський політехнічний інститут". - Харків: НТУ "ХПІ". - 2008. - № 30. - С. 230-231. 3. Никитина Т.Б. Синтез анизотропийного стабилизатора в горизонтальной плоскости / Т.Б. Никитина // Технічна електродинаміка. 2009. - №2. - С. 35-40. 4. Никитина Т.Б. Стохастический синтез цифрового робастного управления многоканальными итерационными системами / Т.Б.Никитина // Електромашинобудування та електрообладнання. - 2008. - № 71. - С.22-27. 5. Никитина Т.Б. Многокритериальный синтез анизотропийного регулятора стабилизатора в вертикальной плоскости / Т.Б. Никитина // Вісник Національного технічного університету "Харківський політехнічний інститут". - Харків: НТУ "ХПІ". - 2009. - № 13. - С. 94-103. 6. Никитина Т.Б. Многокритериальный синтез анизотропийных регуляторов электропривода горизонтального наведения / Т.Б. Никитина // Вісник Кременчуцького державного політехнічного університету. - Кременчуг: КДПУ. - 2009. - № 1/2009 (54). С. 9-12. 7. Никитина Т.Б. Синтез анизотропийного стабилизатора основного вооружения танка в горизонтальной плоскости / Т.Б. Никитина // Механіка та машинобудування. - 2009. - № 2. - С. 135-147. 8. Кузнецов Б.И. Синтез электромеханических систем со сложными кинематическими цепями. - Монография / Б.И. Кузнецов, Т.Б. Никитина, В.В. Коломиец. Харьков: УИПА, 2005. - 512 с. 9. Кузнецов Б.И. Экспериментальное исследование робастного управления главными приводами прокатных станов с учетом их взаимного влияния через прокатываемый металл на стенде двухмассовой электромеханической системы / Б.И.Кузнецов, Т.Б. Никитина, А.В.Волошко, Е.В.Виниченко // Технічна електродинаміка. - 2012. - № 2. - С. 207-212.

Поступила в редакциию 10.09.2013

УДК 62-83:621.77

Розробка та експериментальне дослідження стенду стохастичної двомасової електромеханічної системи / Нікітіна Т.Б., Коломієць В.В., Татарченко М.О., Хоменко В.В. // Вісник НТУ "ХПІ". Серія: Інформатика та моделювання. - Харків: НТУ "ХПІ". - 2013. - № 19 (992). - С. 113 - 120.

Розроблено стенд стохастичної двомасової електромеханічної системи. Проведено експериментальні дослідження динамічних характеристик стенду стохастичної двомасової електромеханічної системи із типовим та робастним регуляторами. Показано, що система із робастним регулятором має меншу помилку порівняно із системою 3 типовим регулятором. Іл.: 6. Бібліогр.: 9 назв.

Ключові слова: стенд стохастичної двомасової електромеханічної системи, експериментальні дослідження, динамічні характеристики.

UDK 62-83:621.77

Development and experimental study of the stand of the stochastic two-mass electromechanical system / Nikitina T., Kolomiets V., Tatarchenko M., Homenko V. // Herald of the National State University "KhPI". Subject issue: Information Science and Modelling. Kharkov: NTU "KhPI". - 2013. - № 19 (992). - P. 113 - 120.

Designed stand stochastic two-mass electromechanical system. Conducted experimental studies of dynamic characteristics of the stand stochastic two-mass electromechanical system with standard and robust controllers. It is shown that a system with robust controller has a lower error than a system with a standard regulator. Figs.: 6. Refs.: 9 titles.

Keywords: stand stochastic two-mass electromechanical system, experimental studies, dynamic characteristics. 